\title{
Streptococcus pneumoniae synergizes with nontypeable Haemophilus influenzae to induce inflammation via upregulating TLR2 Jae Hyang Lim ${ }^{1}$, Unhwan $\mathrm{Ha}^{1}$, Akihiro Sakai ${ }^{2}$, Chang-Hoon Woo ${ }^{3}$, Soo- Mi Kweon ${ }^{2}$, Haidong $\mathrm{Xu}^{1}$ and Jian-Dong $\mathrm{Li}^{* 1}$
}

\author{
Address: ${ }^{1}$ Department of Microbiology and Immunology, University of Rochester Medical Center, Rochester, New York, NY 14642, USA, \\ ${ }^{2}$ Department of Molecular Cell biology, House Ear Institute, Los Angeles, CA 90057, USA and ${ }^{3}$ Cardiovascular Research Institute, University of \\ Rochester Medical Center, Rochester, New York, NY 14642, USA \\ Email: Jae Hyang Lim - Jae-Hyang_Lim@urmc.rochester.edu; Unhwan Ha - Unhwan_Ha@urmc.rochester.edu; \\ Akihiro Sakai - sakai49er@yahoo.co.jp; Chang-Hoon Woo - Changhoon_Woo@urmc.rochester.edu; Soo-Mi Kweon - smkweon@hotmail.com; \\ Haidong Xu - Haidong_Xu@urmc.rochester.edu; Jian-Dong Li* - Jian-Dong_Li@urmc.rochester.edu \\ * Corresponding author
}

Published: 29 July 2008

BMC Immunology 2008, 9:40 doi:10.1 I86/147|-2172-9-40
Received: 14 January 2008

Accepted: 29 July 2008

This article is available from: http://www.biomedcentral.com/147I-2172/9/40

(C) 2008 Lim et al; licensee BioMed Central Ltd.

This is an Open Access article distributed under the terms of the Creative Commons Attribution License (http://creativecommons.org/licenses/by/2.0), which permits unrestricted use, distribution, and reproduction in any medium, provided the original work is properly cited.

\begin{abstract}
Background: Toll-like receptor 2 (TLR2) plays a critical role in mediating inflammatory/immune responses against bacterial pathogens in lung. Streptococcus pneumoniae (S. pneumoniae) and nontypeable Haemophilus influenzae (NTHi) were previously reported to synergize with each other to induce inflammatory responses. Despite the relatively known intracellular signaling pathways involved in the synergistic induction of inflammation, it is still unclear if both bacterial pathogens also synergistically induce expression of surface TLR2.

Results: Here we provide direct evidence that $S$. pneumoniae synergizes with NTHi to upregulate TLR2 expression in lung and middle ear of the mice. Pneumolysin (PLY) appears to be the major virulence factor involved in this synergism. Moreover, S. pneumoniae PLY induces TLR2 expression via a TLR4-MyD88-NF-KB-dependent signaling pathway. Interestingly, tumor suppressor CYLD acts as a negative regulator of $S$. pneumoniae-induced TLR2 up-regulation via negative-crosstalk with NF-kB signaling.
\end{abstract}

Conclusion: Our study thus provides novel insights into the regulation of TLR2 expression in mixed bacterial infections.

\section{Background}

Gram-negative bacterium nontypeable Haemophilus influenzae (NTHi) and gram-positive bacterium Streptococcus pneumoniae (S. pneumoniae) are important bacterial pathogens causing lung infections [1,2]. In children, they cause otitis media (OM), the most common childhood infection and the leading cause of conductive hearing loss [3], while in adults, they exacerbate chronic obstructive pulmonary diseases (COPD), the fourth leading cause of death in the United States $[1,2]$. Although a majority of OM or COPD is mainly associated with a single bacterial pathogen, there is a growing body of evidence that a portion of patients diagnosed with OM or COPD have mixed infections of NTHi and $S$. pneumoniae $[4,5]$. In the mixed infection of NTHi and S. pneumoniae, two bacterial pathogens synergistically induce inflammation through activa- 
tion of specific host signaling pathway $[6,7]$, which is initiated through the recognition of microbial pathogens by means of cell surface receptors.

Toll-like receptors (TLRs) are a class of pathogen recognition receptors that mediate recognition of pathogen-associated molecular pattern. Among 13 mammalian TLRs, TLR2 plays especially a critical role due to its capability of detecting the widest repertoire of pathogen-associated molecular patterns from a large variety of pathogens, including gram-positive or gram-negative bacteria, mycobacteria, fungi, viruses, and parasites [8].

We previously reported that TLR2 is a key receptor recognizing NTHi components [9] and is highly inducible by multiple pro-inflammatory stimuli including NTHi itself [9-12]. Given the fact that $S$. pneumoniae co-exists with NTHi in lung and middle ear infections and synergistically enhances NTHi-induced inflammation [6], it is of particular interest to investigate the molecular mechanism by which TLR2 is likely induced by NTHi and S. pneumoniae in a synergistic manner. In the present study, we showed that $S$. pneumoniae induces TLR2 up-regulation via a cytoplasmic pneumolysin (PLY) through a TLR4MyD88-NF-kB-dependent signaling pathway. Moreover, $S$. pneumoniae induces expression of tumor suppressor cylindromoatosis (CYLD), which in turn negatively regulates $S$. pneumoniae-induced NF- $\mathrm{KB}$ activation and TLR2 expression. These studies thus bring new insights into synergistic regulation of host TLRs in mixed bacterial infections.

\section{Results}

S. pneumoniae pneumolysin induces TLR2 expression in the epithelium of lung and middle ear in vitro and in vivo We initially investigated the effect of $S$. pneumoniae on TLR2 expression in epithelial cells. As shown in Fig. 1A, TLR2 mRNA expression was induced by $S$. pneumoniae treatment in human epithelial HeLa cells in a timedependent manner. TLR2 expression was markedly upregulated at $3 \mathrm{~h}$ after treatment, peaked at $5 \mathrm{~h}$ and declined thereafter. TLR2 up-regulation by $S$. pneumoniae was also confirmed in human respiratory epithelial A549 cells (Fig. 1B). We next determined if TLR2 up-regulation by $S$. pneumoniae occurs at the transcriptional level. As shown in Fig. 1C, transcriptional activity of TLR2 promoter reporter was also up-regulated by $S$. pneumoniae in epithelial cells. Because pneumolysin (PLY) plays a key role in the pathogenesis of $S$. pneumoniae, we investigated whether PLY is a crucial virulence factor for the TLR2 upregulation by $S$. pneumoniae. Human respiratory epithelial cells were treated with either S. pneumoniae WT D39 or pneumolysin-deficient mutant PLN, and mRNA expression level of TLR2 was then measured by Q-PCR analysis. As shown in Fig. 1D, inoculation of $S$. pneumoniae WT
D39 induced TLR2 mRNA expression, whereas S. pneumoniae PLN did not induce it. Moreover, purified PLY induced TLR2 expression to a similar extent to $S$. pneumoniae in middle ear HMEEC-1 epithelial cells (Fig. 1E). To further confirm if $S$. pneumoniae also induces TLR2 expression in vivo, we examined S. pneumoniae- and PLY-induced TLR2 expression in vivo using $S$. pneumoniae-induced pneumonia and OM model in mice. As shown in Fig. 1F \&1G, S. pneumoniae induced TLR2 mRNA expression in both lung and middle ear tissues. Similar results were also observed in PLY-inoculated mice (Fig. 1H). Consistent with our in vitro findings, TLR2 expression was up-regulated by $S$. pneumoniae WT D39 and PLY inoculation but not mutant PLN (Fig. 1H). Furthermore, Western blot analysis confirmed TLR2 up-regulation by $S$. pneumoniae at the protein level after intratracheal inoculation of $S$. pneumoniae in WT mice (Fig. 1I). Taken together, our data showed that $S$. pneumoniae PLY induces TLR2 up-regulation in lung and middle ear in vitro and in vivo.

\section{S. pneumoniae induces TLR2 expression through TLR4- MyD88-NF- $\kappa$ B-dependent signaling pathway}

TLRs play a critical role in recognizing extracellular pathogens and transducing the cell surface interaction between microbes and cells to intracellular components. It was previously reported that cytolysins including Bacillus anthracis anthrolysin O and S. pneumoniae PLY act as TLR4 agonists [13]. Moreover, we reported that PLY triggers TLR4-dependent intracellular signaling pathway in severe $S$. pneumoniae infection in lung $[14,15]$. Thus, we determined whether $S$. pneumoniae induced-TLR2 up-regulation is mediated by TLR4 in vitro and in vivo. Perturbing TLR4 signaling using TLR4 DN mutant plasmid inhibited both $S$. pneumoniae-induced TLR2 promoter activity (Fig. 2A) and TLR2 mRNA expression (Fig. 2B) in vitro. To further investigate whether TLR4 is also critical for S. pneumoniae-induced TLR2 up-regulation in vivo, WT and TLR4 KO mice were intratracheally inoculated with $S$. pneumoniae, and TLR2 mRNA expression was measured in the lungs of inoculated mice. As shown in Fig. 2C, S. pneumoniae induced TLR2 expression in the lungs of WT mice but not TLR4 KO mice in vivo. Because MyD88 is a well-known signaling adaptor for TLR4, we determined whether MyD88 is required for the S. pneumoniae-induced TLR2 expression in vivo. As shown in Fig. 2D, $S$, pneumoniaeinduced TLR2 up-regulation was significantly reduced in MyD88 KO mice compared to WT mice. Having demonstrated a marked decrease in TLR2 expression in TLR4 KO mice, we determined whether TLR4-deficiency caused this decrease via changing bacterial clearance in the lung tissues or inflammatory cell migration into airways. We therefore measured bacteria number from the lungs of $S$. pneumoniae-inoculated WT and TLR4 KO mice $24 \mathrm{hrs}$ after inoculation and also measured inflammatory cell migration into airway from the BAL fluid of vehicle- or S. pneu- 


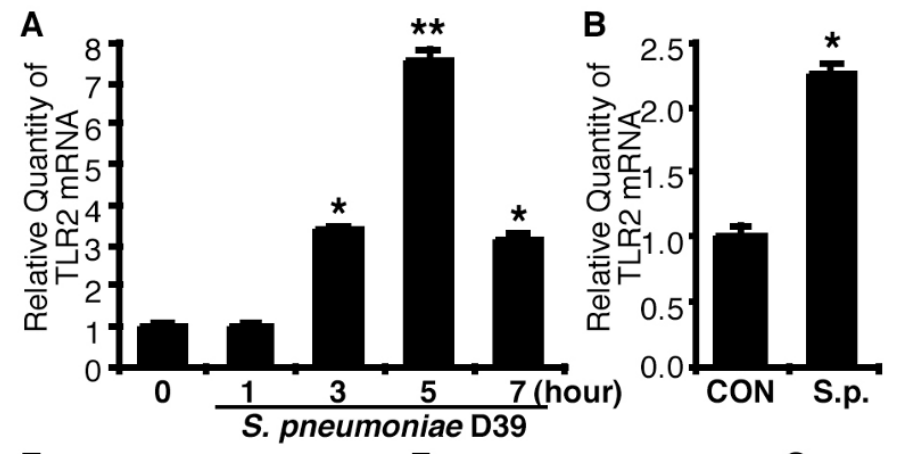

E

F Lung (in vivo)

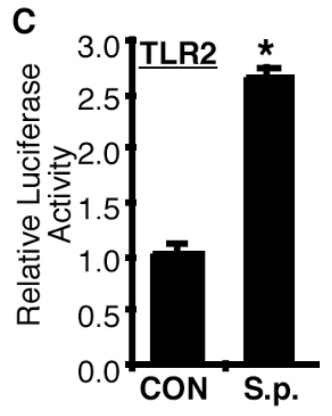

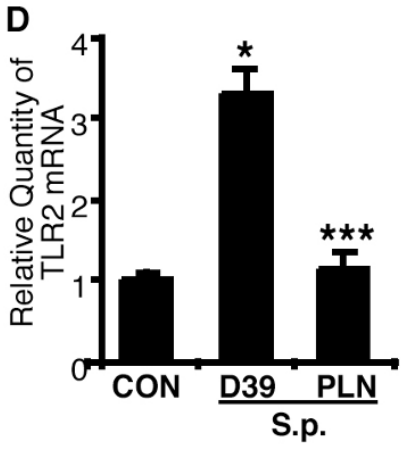

H Lung (in vivo)

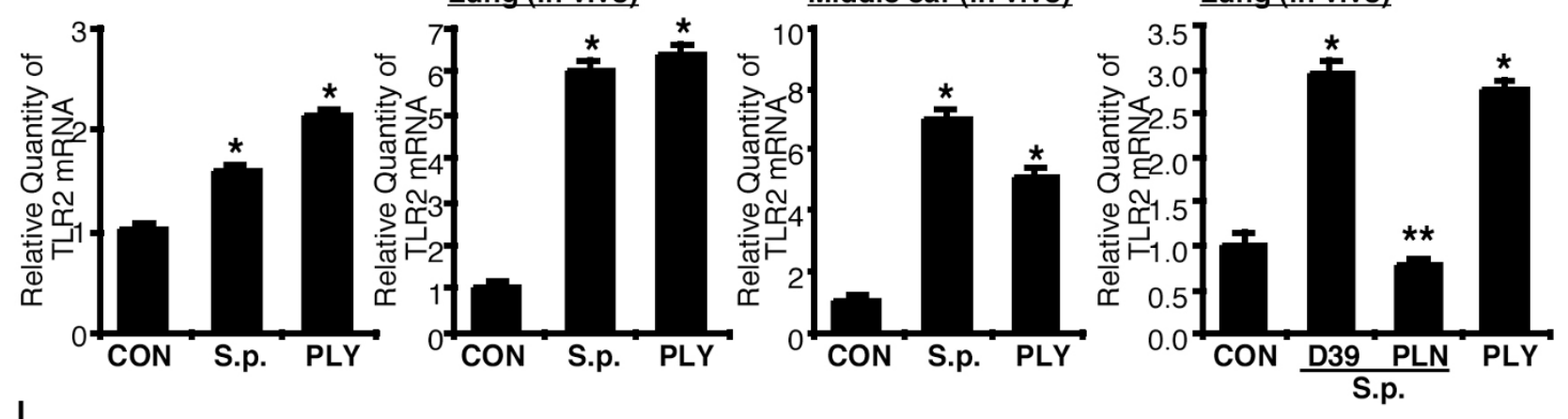

I
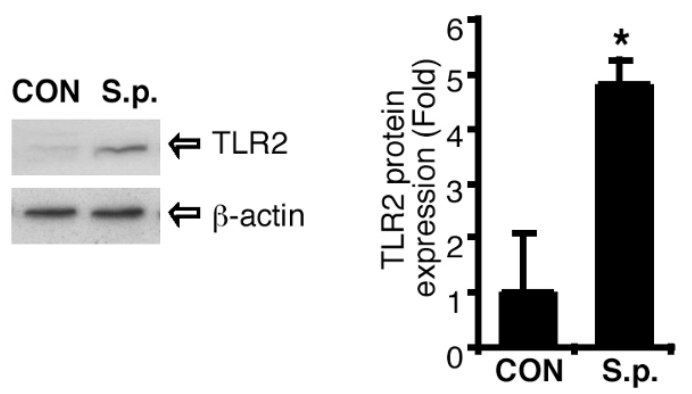

Figure I

S. pneumoniae induces TLR2 up-regulation via pneumolysin in the epithelium of lung and middle ear. (A) Time dependence of TLR2 up-regulation in response to S. pneumoniae (S.p.). HeLa cells were treated with S.p. for I, 3, 5 and 7 hours, and relative quantity of TLR2 mRNA expression was measured by Q-PCR analysis. (B)S.p.-induced TLR2 mRNA expression in lung epithelial cell A549. Cells were treated with S.p., and relative quantity of TLR2 mRNA expression was measured by QPCR analysis 5 hours after treatment. (C) S.p.-induced TLR2 promoter activity. HeLa cells were transiently transfected with TLR2 luciferase reporter gene, treated with S.p., and relative luciferase activity was measured 5 hours after treatment. (D) The effects of S.p. WT strain D39 and PLY-deficient strain PLN on S.p.-induced TLR2 expression. Cells were treated either with S.p. D39 or PLN, and mRNA expression of TLR2 was measured by Q-PCR analysis 3 hours after treatment. (E) S.p.- and PLYinduced TLR2 mRNA expression in middle ear cell HMEEC-I. Cells were treated with S.p. or PLY, and TLR2 mRNA expression was measured 5 hours after treatment by Q-PCR analysis. (F \& G) S.p.- and PLY-induced TLR2 expressions in the lung and middle ear of the mice. Wild-type mice were intratracheally inoculated with S.p. or PLY for S.p.-induced pneumonia (F) and inoculated into the middle ear through tympanic membrane for S.p.-induced otitis media model (G). Relative quantity of TLR2 mRNA was measured from the lungs of inoculated mice 3 hours after inoculation and from the middle ears of inoculated mice 9 hours after inoculation. $(\mathrm{H})$ The effect of PLY on S.p.-induced TLR2 expression was measured using S.p. WT strain D39 and PLY-deficient strain PLN in mice in vivo. WT mice were intratracheally inoculated with S.p. D39 or PLN, or purified PLY, and TLR2 mRNA expression was measured from the lungs of inoculated mice 9 hours after inoculation by Q-PCR analysis. (I) Protein expression of TLR2 is up-regulated following S.p. infection. TLR2 protein expression was measured from the lungs WT mice inoculated with S.p. by western blotting analysis 6 hours after inoculation (left panel), and band data was analyzed using Kodak Image analysis system and data was presented as Fold induction (right panel). Data are means \pm S.D. $(n=3)$. *, $p<0.05$ compared with CON, **, $p<0.01$ compared with CON, ***, $p<0.05$ compared with S.p. D39, CON, control. 

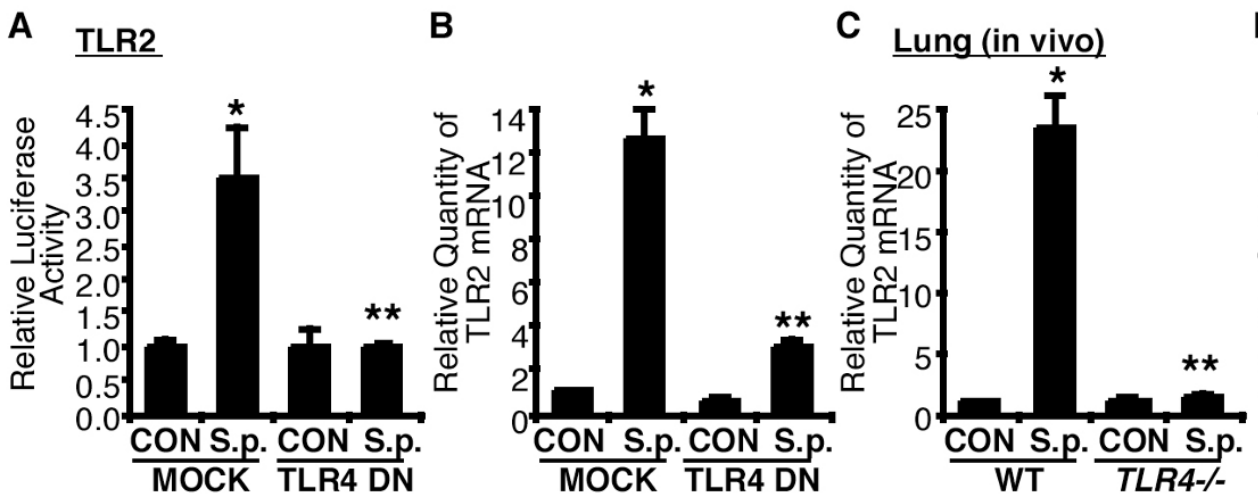

\section{Lung (in vivo)}
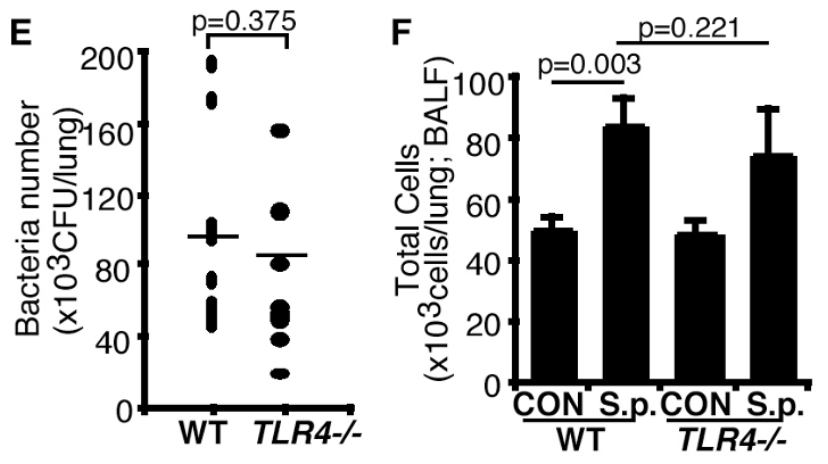

G
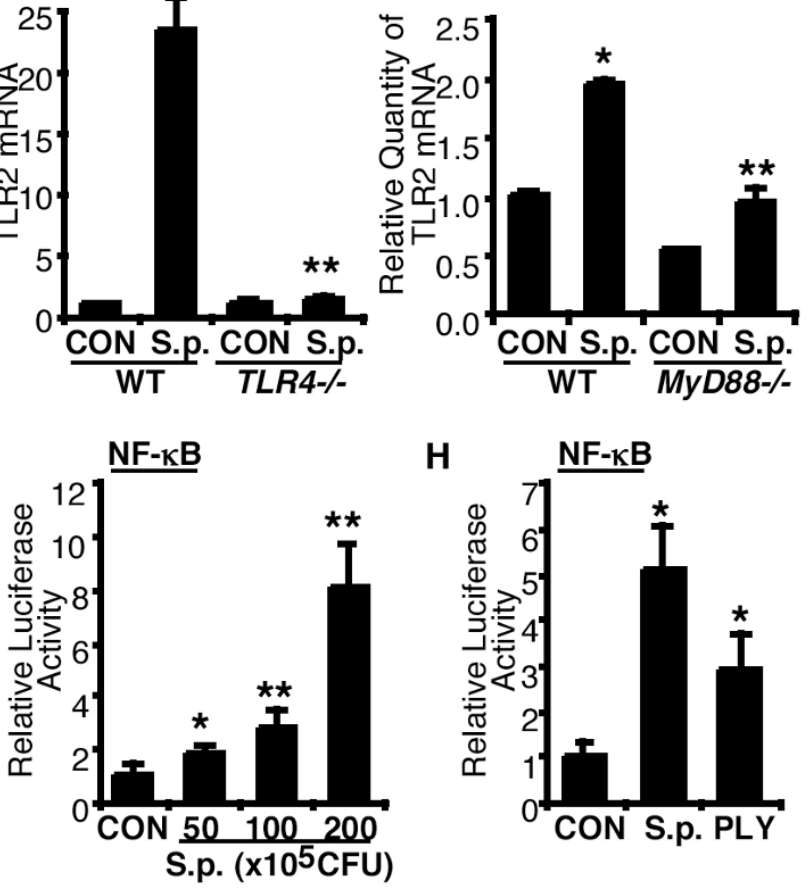

I

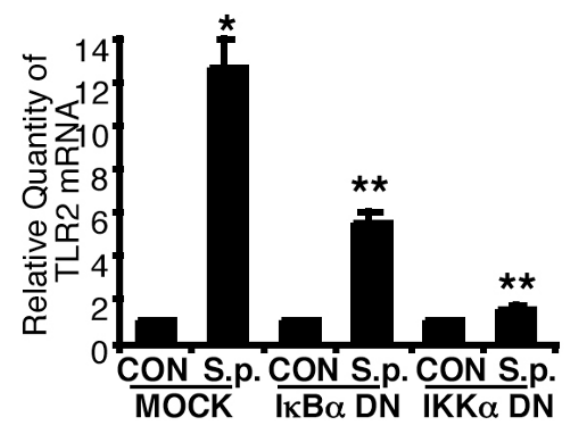

$\mathbf{J}$

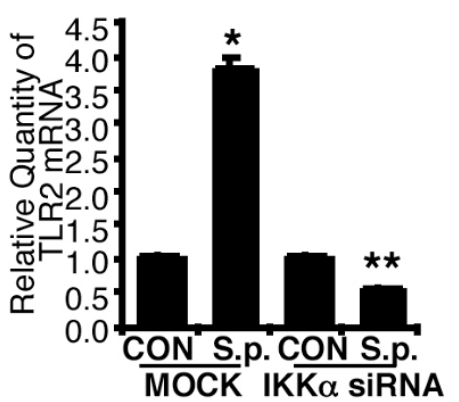

H

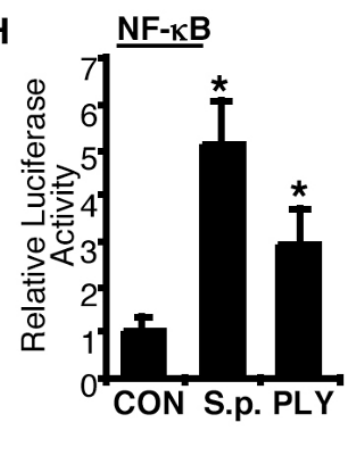

Figure 2

TLR4-MyD88-NF- $K$ B signaling pathway mediates S. pneumoniae-induced TLR2 up-regulation. (A) Cells were transiently transfected with TLR2 promoter reporter gene with TLR4 DN mutant plasmid or empty vector, and S.p.-induced luciferase activity was measured 5 hours after S.p. treatment. (B) Cells were transiently transfected either with TLR4 DN mutant plasmid or empty vector, and S.p.-induced TLR2 mRNA expression was measured by Q-PCR analysis. (C) WT and TLR4 KO mice were intratracheally inoculated with S.p., and TLR2 mRNA expression was measured from the lungs of inoculated mice by Q-PCR analysis. (D) WT and MyD88 KO mice were intratracheally inoculated with S.p., and TLR2 mRNA expression was measured from the lungs of inoculated mice by Q-PCR analysis. (E) Bacterial number in the lungs of WT and TLR4 KO mice following S.p. inoculation. WT and TLR4 KO mice were intratracheally inoculated with I $\times 10^{7}$ CFU of S.p. D39, and CFU of the lungs were measured 24 hours after inoculation. (F) Inflammatory cell migration into airway in WT and TLR4 KO mice following S.p. inoculation. WT and TLR4 KO mice were intratracheally inoculated with I $\times 10^{7} \mathrm{CFU}$ of S.p., and total cells were measured from the BAL fluid of the lungs of saline- or S.p.-inoculated mice 6 hours after inoculation. $(G \& H)$ Cells were transfected with NF- $\kappa B$ reporter gene, and S.p.- or PLY-induced NF- $\kappa B$ promoter activity was measured as relative luciferase activity. (I \& J) Cells were transiently transfected with IKB $\alpha \mathrm{DN}$, IKK $\alpha \mathrm{DN}$, IKK $\alpha$ siRNA, empty vector, or control siRNA, and S.p.induced TLR2 mRNA expression was measured by Q-PCR analysis. Data in A-D \& G-J are means \pm S.D. $(n=3)$. *, $p<0.05$ compared with CON, **, $p<0.05$ compared with S.p. in MOCK or WT mice, CON, control inoculated with vehicle. 
moniae-inoculated WT and TLR4 KO mice 6 hrs after inoculation (Fig. 2E \&2F). Neither bacteria number of the lungs nor inflammatory cell migration into airways in TLR4 KO mice was significantly different from those in WT mice. The results reported herein demonstrate that, under the conditions reported here, TLR4-deficiency does not affect the bacterial clearance of $S$. pneumoniae and inflammatory cell migration response to $S$. pneumoniae, and decrease in S. pneumoniae-induced TLR2 expression in TLR4 KO mice is independent of both bacteria number and inflammatory cells in the lung in vivo. Collectively, our data suggest that $S$. pneumoniae induces TLR2 up-regulation via TLR4-MyD88 signaling pathway.

Because NF- $\kappa$ B plays an important role in mediating PLYinduced host responses, we next determined if $N F-\kappa B$ is also involved in mediating TLR2 up-regulation by PLY $[9,10,16]$. As shown in Fig. $2 \mathrm{G} \& 2 \mathrm{H}, \mathrm{S}$. pneumoniae induced NF- $\kappa \mathrm{B}$ activation in a dose-dependent manner. Similar result was also observed in PLY-treated cells. We further determined the involvement of IKK $\alpha$-I $\kappa \mathrm{B} \alpha$, the key signaling pathway upstream of NF- $\kappa \mathrm{B}$, in TLR2 induction $[6,14]$. As shown in Fig. 2I, perturbing IKK $\alpha$ and I $\mathrm{B} \alpha$ signaling by overexpressing DN mutant plasmid inhibited $S$. pneumoniae-induced TLR2 mRNA up-regulation. This finding was further confirmed by using IKKosiRNA (Fig. 2J). Thus it is clear that $S$. pneumoniae-induced TLR2 upregulation is mediated by IKK $\alpha$ and I $\mathrm{I} B \alpha$-dependent NF$\kappa \mathrm{B}$ activation.

\section{S. pneumoniae induced-TLR2 up-regulation is negatively regulated by tumor suppressor CYLD}

Bacteria modulates many innate immune responses via induction of negative regulatory molecules $[15,17,18]$. We previously reported that $S$. pneumoniae induces tumor suppressor CYLD, an important negative regulator of NF$\kappa \mathrm{B}$ signaling pathway, in severe $S$. pneumoniae infection [15]. NTHi induced CYLD expression, which in turn leads to negative regulation of NTHi-induced NF- $\mathrm{B}$-dependent inflammation [19]. CYLD expression is relatively low in lung but is highly induced under diseased condition [18]. To determine the role of CYLD in S. pneumoniae-induced TLR2 expression in the lung and middle ear, we first evaluated up-regulation of CYLD in vitro. As shown in Fig. 3A, S. pneumoniae induced CYLD expression not only in lung epithelia cell A549, but also in middle ear epithelial cell HMEEC-1. To further confirm whether $S$. pneumoniae induces CYLD both in the lung and middle ear in vivo, WT mice were intratracheally or transtympanically inoculated with S. pneumoniae, and CYLD mRNA expression was then measured in the lung and middle ear tissues of inoculated mice, respectively. As shown in Fig. 3B, S. pneumoniae induced CYLD expression in both lung and middle ear of the mice.
To further investigate the role of CYLD in S. pneumoniaeinduced NF- $\kappa \mathrm{B}$ activation, the effect of CYLD knockdown using CYLD siRNA was measured. As shown in Fig. 3C. $S$. pneumoniae- and PLY-induced NF- $\kappa \mathrm{B}$ promoter activity was greatly enhanced by CYLD siRNA. To investigate whether CYLD acts as a negative regulator for S. pneumoniae-induced TLR2 expression, the effects of CYLD siRNA on $S$. pneumoniae-induced TLR2 mRNA expression were measured. As shown in Fig. 3D, knockdown of CYLD expression using CYLD siRNA greatly enhanced $S$. pneumoniae-induced TLR2 mRNA expression. To determine the in vivo role of CYLD on S. pneumoniae-induced TLR2 expression, we measured TLR2 mRNA expression in lung tissues of WT and CYLD KO mice after S. pneumoniae inoculation. Consistent with our in vitro findings, S. pneumoniae-induced TLR2 mRNA up-regulation was enhanced in CYLD KO mice (Fig. 3E). Together, our data indicate that CYLD acts as a negative regulator for $S$. pneumoniaeinduced NF- $\kappa \mathrm{B}$ activation and TLR2 up-regulation.

\section{S. pneumoniae synergizes with NTHi to induce inflammatory response via TLR2}

To determine whether $S$. pneumoniae synergizes with NTHi to induce inflammatory response, we investigated the effect of $S$. pneumoniae and NTHi on the expression of IL-1 $\beta$, a key NF- $\kappa \mathrm{B}$-dependent pro-inflammatory cytokine in lung and middle ear. As shown in Fig. 4A, S. pneumoniae and NTHi synergistically enhanced expression of IL-1 $\beta$ ? mRNA in human lung epithelial cell. Moreover, S. pneumoniae and NTHi also synergistically induced expression of IL-1 $\beta$ mRNA in the lungs of mice (Fig. 4B). Next we investigated the role of TLR2 in synergistic induction of NF-кB-dependent inflammatory response by $S$. pneumoniae and NTHi. As shown in Fig. 4C, siRNA mediated knockdown of TLR2 markedly inhibited synergistic induction of IL-1 $\beta$ mRNA expression by $S$. pneumoniae and NTHi, thereby providing evidence for the involvement of TLR2 in this synergistic induction. This finding was further confirmed in the cells from TLR2 KO mice in vitro (Fig. 4D). Having shown that S. pneumoniae-induced TLR2 up-regulation is inhibited by TLR4-deficiency, and TLR2 up-regulation is closely associated with synergistic response by $S$. pneumoniae with $\mathrm{NTHi}$, we determined if TLR4-deficiency affects synergistic activation of NF- $\kappa B$ and subsequent gene expression by $S$. pneumoniae with NTHi. We first measured NF- $\kappa \mathrm{B}$ activation and mRNA expressions of TLR2 and IL- $1 \beta$ following co-treatment with S. pneumoniae and NTHi in cells (Fig. 4E) and lung tissues (Fig. 4F \&4G) of WT and TLR4 KO mice. As shown in Fig. $4 \mathrm{E}, \mathrm{NF}-\kappa \mathrm{B}$ activation by $S$. pneumoniae with or without NTHi was significantly decreased in the cells from TLR4 KO mice compared with those in cells from WT mice (Fig. 4E). In addition, mRNA expressions of TLR2 and IL$1 \beta$ by $S$. pneumoniae with or without NTHi in the lung tissues of TLR4 KO mice were also significantly decreased 
A

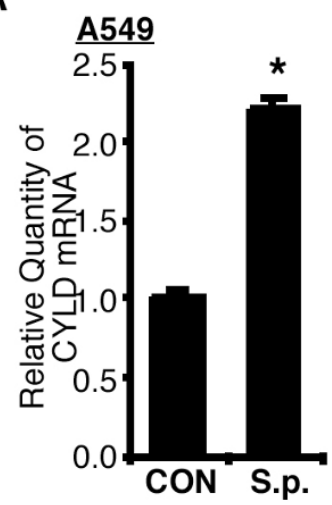

C

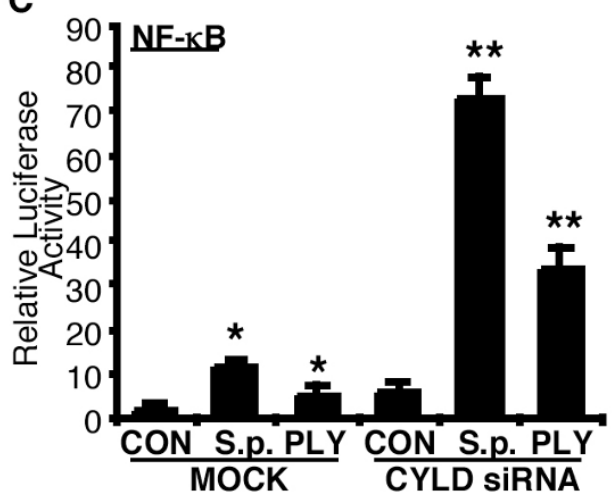

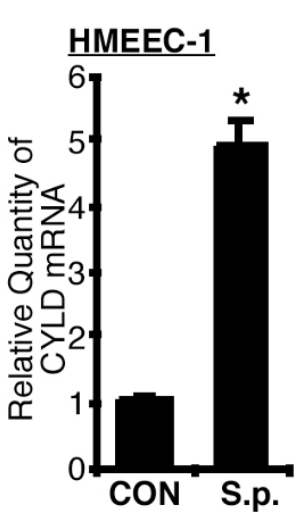

D

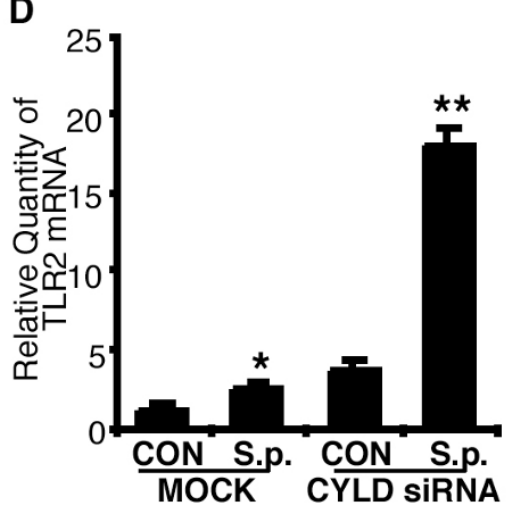

B

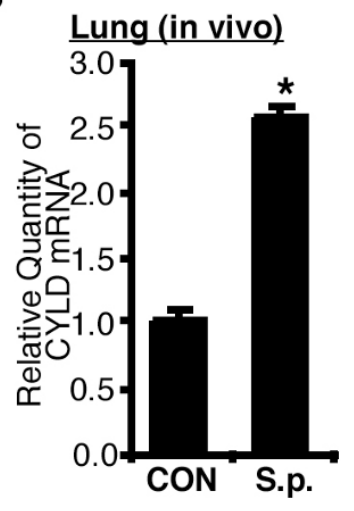

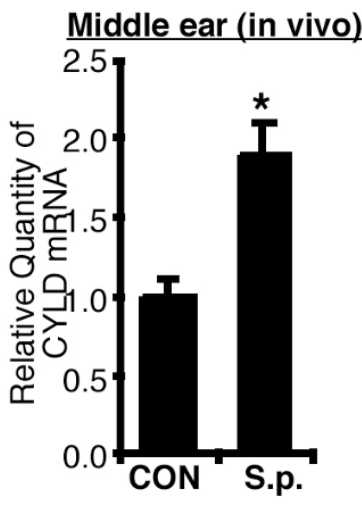

E

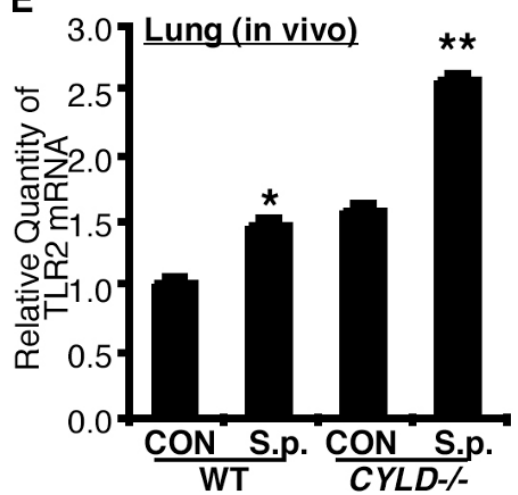

Figure 3

CYLD negatively regulates S. pneumoniae-induced TLR2 up-regulation. (A) Human alveolar epithelial cell A549 and human middle ear epithelial cell HMEEC-I were treated with S.p., and CYLD mRNA expression was measured 5 hours after treatment by Q-PCR analysis. (B) WT mice were intratracheally or transtympanically inoculated with S.p. for pneumonia and otitis media model, respectively, and CYLD mRNA expression was measured from the lungs and middle ear tissues of inoculated mice 3 hours after inoculation and 9 hours after inoculation, respectively. Data in A \& B are means \pm S.D. $(n=3)$. *, $p<$ 0.05 compared with CON, CON, control treated with vehicle. (C) Cells were transfected with NF-KB reporter gene with CYLD siRNA or empty vector. S.p.- or PLY-induced NF-KB promoter activity was measured as relative luciferase activity. (D) Cells were transfected either with CYLD siRNA or empty vector, and S.p.-induced TLR2 mRNA expression was measured 5 hours after treatment by Q-PCR analysis. (E) WT and CYLD KO mice were intratracheally inoculated with S.p., and TLR2 mRNA expression was measured from the lungs of inoculated mice 6 hours after inoculation by Q-PCR analysis. Data are means \pm S.D. $(n=3)$. *, $p<0.05$ compared with CON, **, $p<0.05$ compared with S.p. in MOCK (A-C) or in WT mice (D), $\mathrm{CON}$, control inoculated with vehicle, MOCK, transfected with empty vector.

compared with those in WT mice (Fig. 4F \&4G). Taken together, these findings suggest that $S$. pneumoniae synergizes with NTHi to induce NF- $\mathrm{BB}$ activation and subsequent gene expression via TLR2 up-regulation.

\section{Discussion}

There is a growing body of evidence showing that a portion of patients diagnosed with OM or COPD have mixed infections of NTHi and S. pneumoniae [4,5]. Under in vivo situations such as polymicrobial infections, mucosal epithelial surfaces are simultaneously exposed to a variety of bacteria and bacterial components, and one microbe synergizes with another one to induce the inflammatory response by activating intracellular signaling pathway $[6,7]$. Among many other innate immune components, TLRs are critical for initiating and mediating immune response. Thus, regulation of TLRs expression plays an important role in modulating host response to microbes. However, little is known about the molecular mechanisms underlying regulation of TLRs expression in mixed infection.

As we previously reported, TLR2 expression is relatively low in unstimulated epithelial cells but is markedly upregulated in response to invading microbes $[10,11]$. However, the link between TLR2 up-regulation and mixed 


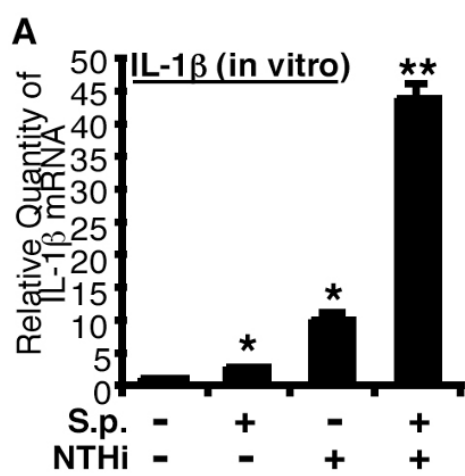

D
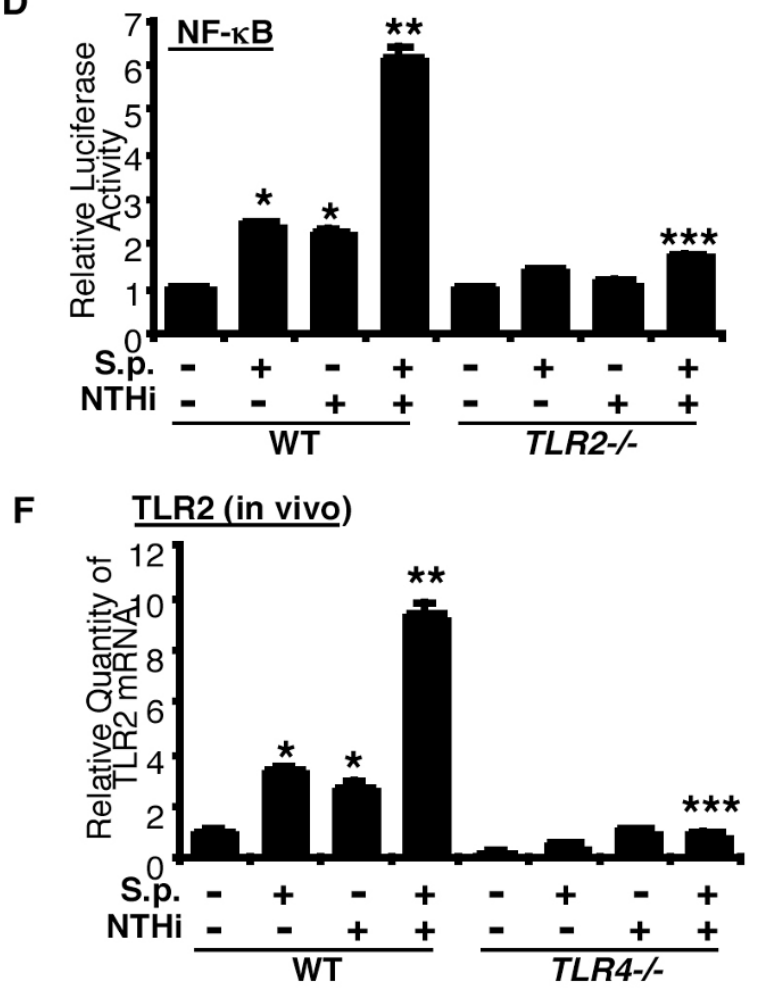

B
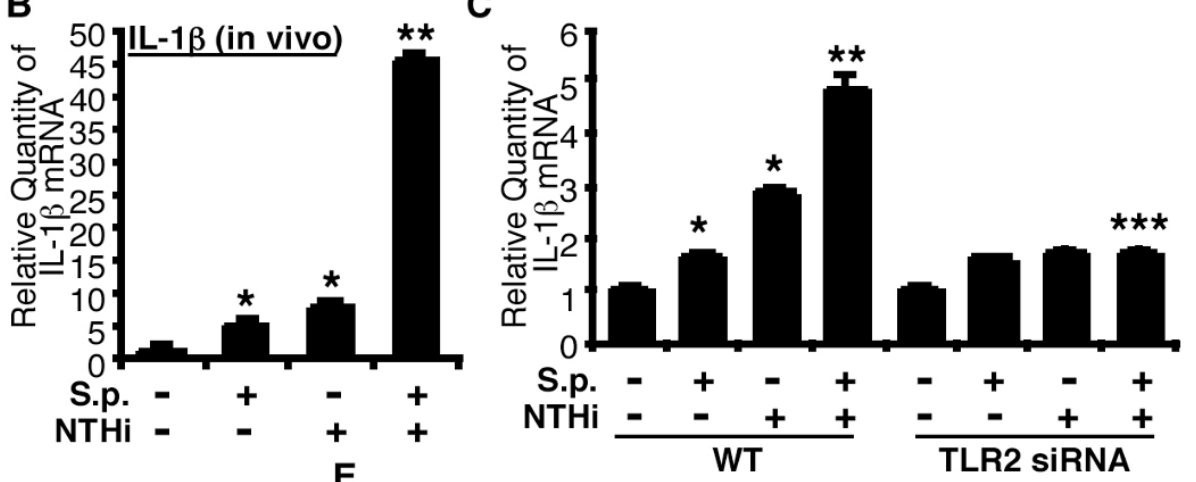

E

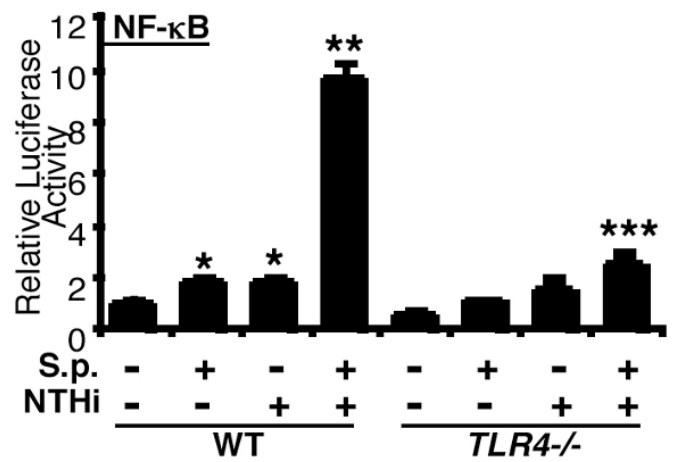

G

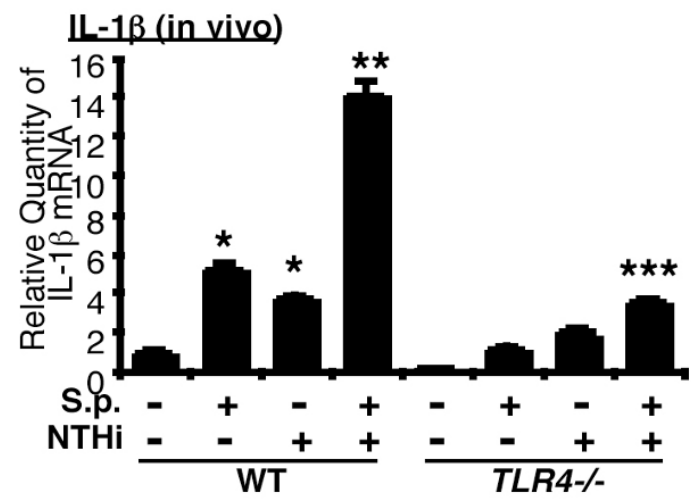

Figure 4

S. pneumoniae synergizes with NTHi to induce inflammatory response by up-regulating TLR2 expression. (A) HeLa cells were treated with S.p. with or without NTHi, and IL-I $\beta$ mRNA expression was measured 5 hours after treatment by Q-PCR analysis. (B) WT mice were intratracheally inoculated with S.p. with or without NTHi, and IL-I $\beta$ mRNA expression was measured from the lungs of inoculated mice 6 hours after inoculation by Q-PCR analysis. (C) Cells were transfected with TLR2 siRNA or control siRNA, treated with S.p. with or without NTHi, and IL-I $\beta$ mRNA expression was measured 5 hours after infection by Q-PCR analysis. (D) NF- $\kappa$ B luciferase activity following S.p., NTHi, or S.p. with NTHi in MEF cells from WT and TLR2 KO mice. MEF cells from WT and TLR2 KO mice were transfected with NF- $\kappa B$ reporter gene, inoculated with S.p., $\mathrm{NTHi}$, or S.p. with NTHi, and relative luciferase activity was measured 5 hours after inoculation. (E) NF- $\kappa \mathrm{B}$ luciferase activity following S.p., NTHi, or S.p. with NTHi in MEF cells from WT and TLR4 KO mice. MEF cells from WT and TLR4 KO mice were transfected with NF-kB reporter gene, inoculated with S.p., NTHi, or S.p. with NTHi, and relative luciferase activity was measured 5 hours after inoculation. ( $F \& G)$ TLR2 and IL-I $\beta$ up-regulation in the lungs of WT and TLR4 KO mice following S.p., $\mathrm{NTHi}$, or S.p. with NTHi in vivo. WT and TLR4 KO mice were intratracheally inoculated with S.p., NTHi, or S.p. with NTHi, and TLR2 (F) and IL-I $\beta(G)$ mRNA expression was measured from the lung of inoculated mice 6 hours after inoculation. Data are means \pm SD $(n=3)$. *, $p<0.05$ compared with control, **, $p<0.05$ compared with NTHi treatment, ***, $p<0.05$ compared with S.p. with NTHi treatment in MOCK or WT mice. 
infection in otitis media and pneumonia still remains unknown. From what we have shown in the present data, it is evident that TLR2 expression is up-regulated by $S$. pneumoniae via a TLR4-MyD88-NF- $\mathrm{BB}$-dependent signaling pathway through its well-known virulence factor PLY. We further show that $S$. pneumoniae and NTHi synergize with each to induce NF- $\mathrm{KB}$ activation and IL- $1 \beta$ expression dependently of TLR 2 up-regulation. In the present study, we provided evidence for the first time that $S$. pneumoniae synergizes with NTHi to induce NF-кB-dependent inflammatory response likely via TLR2 up-regulation in lung and middle ear epithelia cells, thereby bringing new insight into our understanding of the complex regulatory mechanisms underlying inflammation in polymicrobial infections.

In addition to TLR2 and TLR4, TLR1, 6, and 9 have also been shown to be involved in $S$. pneumoniae infection [2022]. Therefore we evaluated lung epithelial cell expression of 10 TLRs (TLR1-10) and CD14, MyD88, MD1 and 2 following $S$. pneumoniae treatment in A549 cells. In untreated cells, the expression level of TLR1, 3, 4, 5, 6 and 9 was relatively high compared to those of TLR2, 7, and 8 . Interestingly, the expression of TLR2, 7 and 8 at the mRNA level was highly up-regulated following $S$. pneumoniae treatment (Data not shown), implying a possible important role for TLR2 up-regulation in bacterial infections. Indeed, the data we presented in this study demonstrated that the synergistic activation of NF- $\mathrm{\kappa B}$ and inflammation by $S$. pneumoniae and NTHi is dependent on TLR2 up-regulation.

Although there is strong evidence to support that TLR4 is a critical receptor for recognizing pneumolysin and pneumolysin is a major virulence factor of pneumococcal infection, the role of TLR4 in pneumococcal infection is not fully understood [23-26]. It remains somewhat controversial whether TLR4-deficiency confers susceptibility or tolerance to pneumococcal infection in vivo $[23,24,27,28]$. Controversial phenotype of TLR4-deficiency mice in $S$. pneumoniae infection may be partly due to the strain differences of the mice used in each experiment, e.g. using $\mathrm{C} 3 \mathrm{H} / \mathrm{HeJ}, \mathrm{BALB} / \mathrm{C}$ and $\mathrm{C} 57 \mathrm{BL} / 6$ as background strains. It is well known that different mouse strains exhibit a very different susceptibility to pneumococcal infection [28-30]. BALB/c mice, which were used as a background strain of TLR4 KO mice in our current experiment, were known as resistant strain in pneumococcal infection compared to other strains [30]. TLR4-deficiency in this strain thus may have no significant effect on pneumococcal clearance.

Another interesting finding in this study is the experimental evidence for the negative regulation of $S$. pneumoniaeinduced TLR2 up-regulation by tumor suppressor CYLD.
CYLD was previously identified as an important regulator not only in tumor development $[31,32]$ but also in adaptive immune response [33,34]. Recent evidence demonstrated that CYLD also serves as a key regulator for innate immune response via negative-crosstalk with MAPKs and NF- $\kappa B ~[15,17,19,32]$. Moreover we recently identified an important role for CYLD in modulating host antiviral response by regulating TLR7 expression in mixed infection of bacteria and virus [35]. However, still unknown is how CYLD regulates inflammatory response in mixed bacterial infection.

\section{Conclusion}

In the present study we showed for the first time that $S$. pneumoniae synergizes with NTHi to induce NF- $\mathrm{BB}$ activation and the subsequent inflammatory response via upregulation of TLR2. Moreover, we showed that $S$. pneumoniae-induced TLR2 up-regulation is mediated by TLR4MyD88-IKK $\alpha$-I $\mathrm{B} \alpha$ signaling pathway. Interestingly, tumor suppressor CYLD acts as a negative regulator for the synergistic induction of inflammation by $S$. pneumoniae and NTHi, thereby implying a critical role for CYLD in preventing overactive inflammatory response in mixed infections. This study may bring new insights into the molecular mechanisms underlying polymicrobial infections, especially mixed infection of gram-positive and negative bacteria.

\section{Methods \\ Cell cultures}

Human alveolar epithelial cell line A549, human middle ear epithelia cell line HMEEC-1 derived by human papillomavirus immortalization of primary human middle ear epithelial cell, and human cervix epithelia cell line HeLa were maintained as described previously [6,9-12]. Mouse embryonic fibroblasts (MEFs) were isolated from E13 embryos of WT, TLR2 KO and TLR4 KO mice and cultured in DMEM.

\section{Bacteria and pneumolysin}

Clinical isolates of S. pneumoniae WT D39, and D39 isogenic PLY-deficient mutant PLN were used in both in vitro and in vivo experiments [36]. S. pneumoniae was grown on chocolate agar plates and in Todd-Hewitt broth supplemented with $0.5 \%$ yeast extract (THY) at $37^{\circ} \mathrm{C}$ in a humidified $5 \% \mathrm{CO}_{2}$ water-jacketed incubator without shaking. Mid-log phase bacterial culture was used in the experiments. S. pneumoniae was inoculated at the concentration of $1 \times 10^{6}$ or $1 \times 10^{7}$ colony forming unit (CFU) in both in vitro and in vivo experiments unless otherwise specifically indicated in the figures and figure legends. Native PLY was described previously [25] and treated/inoculated at the concentration of $100 \mathrm{ng} / \mathrm{ml}$. NTHi strain 12, a clinical isolate, was used in this study. NTHi was inoculated at the concentration of $1 \times 10^{7} \mathrm{CFU}$ in both in vitro and in 
vivo experiments. The preparation of bacterial lysate was described previously $[10,14]$.

\section{Animal and animal experiments}

C57BL/ 6 and BALB/c mice were purchased from Charles River. TLR4 KO mice were purchased from Jackson Lab., and TLR2 and MyD88 KO mice were kindly provided by Dr. S. Akira. CYLD KO mice were reported previously [15].

For the S. pneumoniae or PLY-induced pneumonia model in mice, animals were intratracheally inoculated with $1 \times$ $10^{7} \mathrm{CFU}$ of $\mathrm{S}$. pneumoniae or $100 \mathrm{ng}$ of PLY in $50 \mu \mathrm{l}$ of saline. At the time point indicated in the figure legends, mice were sacrificed by overdose injection of sodium pentobarbital, and mRNA expressions of TLR2, CYLD, and IL-1 $\beta$ were measured in the lung tissues of control and S. pneumoniae or PLY-inoculated mice as described below. For the $S$. pneumoniae or PLY-induced OM model in mice, animals were inoculated with $S$. pneumoniae or PLY in $10 \mu$ l of saline via transtympanic inoculation. Nine hours after inoculation, animals were sacrificed by overdose injection of sodium pentobarbital, and middle ears were dissected from the skull for extraction of mRNA for measuring TLR2 and CYLD expression. For the control inoculation in both pneumonia and OM model, $50 \mu \mathrm{l}$ or $10 \mu \mathrm{l}$ of saline was inoculated, respectively. All animal experiments were approved by the Institutional Animal Care and Use Committee at University of Rochester.

\section{Transfection and small interfering RNA (siRNA)}

The expression plasmids including TLR4 dominant-negative (DN) mutant, I $\mathrm{DB} \alpha \mathrm{DN}$ (S32/36A), IKK $\alpha$ DN (K44M), and NF- $\mathrm{B}$ and TLR2 luciferase reporters were described previously [6,9-12]. RNA-mediated interference for down-regulating CYLD, IKK $\alpha$, and TLR2 expression was conducted using small interfering CYLD siRNA (pSuper-CYLD), IKKasiRNA (Dharmacon), and TLR2 siRNA (Dharmacon) as described previously [17]. All transient transfections of plasmids were carried out using TransITLT1 (Mirus), and RNA-mediated interferences were carried out using Lipofectamine ${ }^{\mathrm{TM}} 2000$ (Invitrogen) following the manufacturer's instructions.

\section{Real-time quantitative PCR (Q-PCR) analysis}

Q-PCR analysis of TLR2, CYLD, and IL- $1 \beta$ was conducted as follows. Total RNA was isolated from cells and lung and middle ear tissues of mice using TRIzol (Invitrogen) following the manufacturer's instruction. RT reaction was conducted using TaqMan reverse transcription reagents (ABI) following the manufacturer's instruction. PCR amplification was performed with TaqMan universal master mix (ABI) for TLR2 and IL-1 $\beta$ and SYBR green universal master mix (ABI) for CYLD. Predeveloped human and mouse TLR2 and IL-1 $\beta$ Q-PCR primers were purchased from $\mathrm{ABI}$, and primer sequences for human and mouse
CYLD are as follows: human CYLD sense, 5 '-ttc agc ctg ttt aaa aac aga aa-3'; human CYLD antisense, 5'-tcc cca gga cct gcg taa t-3'; mouse CYLD sense, 5'-ctc agc cta ttt aga aac aga ct-3'; mouse CYLD antisense, 5'-tct cct ggg cct gca aat3'. Reactions were amplified and quantified using an ABI 7500 sequence detector and manufacturer's software (ABI). The relative quantity of TLR2, CYLD, and IL-1 $\beta$ mRNA was obtained using the comparative CT method and was normalized using predeveloped TaqMan assay reagent human cyclophilin and mouse GAPDH as an endogenous control for human mRNA and mouse mRNA, respectively.

\section{Western Blotting analysis}

Antibody against TLR2 was purchased from eBioscience, and Western blot analysis of TLR2 was conducted as follows. WT mice were first intratracheally inoculated with 1 $\times 10^{7} \mathrm{CFU}$ of $S$. pneumoniae and total protein was then extracted from the lung tissues of saline- or S. pneumoniaeinoculated mice 6 hours after inoculation. Western blot analysis was performed as described previously $[10,14,37]$.

\section{Statistical analysis}

Statistical analysis was performed with student t-test. $P$ values of less than 0.05 were considered statistically significant.

\section{Abbreviations}

S. pneumoniae: Streptococcus pneumoniae; NTHi: nontypeable Haemophilus influenza; PLY: pneumolysin; TLR: Toll-

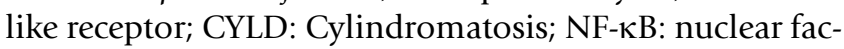
tor-kappa B; WT: wild-type; KO: knock-out; Q-PCR: realtime quantitative RT-PCR.

\section{Authors' contributions}

The authors contributed to the work as following: JHL designed and performed experiments, collected and analyzed data, and prepared manuscript; UH and AS designed research and performed experiment; CHW and HX analyzed the data and contributed to the preparation of the manuscript; SKW performed experiments; JDL conceived study, designed research, analyzed data, and wrote the manuscript. All authors read and approved the final manuscript.

\section{Acknowledgements}

We are grateful to Drs. R. Malley and A. Srivastava, R. Bernards and S. Akira for kindly providing various reagents. This work was supported by grants from National Institute of Health DC004562 and DC005843 (to J.-D. Li).

\section{References}

I. Sethi S, Evans N, Grant BJ, Murphy TF: New strains of bacteria and exacerbations of chronic obstructive pulmonary disease. N Engl J Med 2002, 347:465-47I.

2. Future directions for research on diseases of the lung. American Thoracic Society. Medical Section of the American 
Lung Association. This report was approved by the ATS Board of Directors in November 1994. Am J Respir Crit Care Med 1995, 152:1713-1735.

3. Klein JO: What's new in the diagnosis and management of otitis media? Pediatr Ann 2002, 31 :777-778.

4. Gok U, Bulut Y, Keles E, Yalcin S, Doymaz MZ: Bacteriological and PCR analysis of clinical material aspirated from otitis media with effusions. Int J Pediatr Otorhinolaryngol 200I, 60:49-54.

5. Ozyilmaz E, Akan OA, Gulhan M, Ahmed K, Nagatake T: Major bacteria of community-acquired respiratory tract infections in Turkey. Jpn J Infect Dis 2005, 58:50-52.

6. Kweon SM, Wang B, Rixter D, Lim JH, Koga T, Ishinaga $H$, Chen LF, Jono H, Xu H, Li JD: Synergistic activation of NF-kappaB by nontypeable $H$. influenzae and $S$. pneumoniae is mediated by CK2, IKKbeta-IkappaBalpha, and p38 MAPK. Biochem Biophys Res Commun 2006, 35 I:368-375.

7. Ratner AJ, Lysenko ES, Paul MN, Weiser JN: Synergistic proinflammatory responses induced by polymicrobial colonization of epithelial surfaces. Proc Natl Acad Sci U S A 2005, 102:3429-3434.

8. Texereau J, Chiche JD, Taylor W, Choukroun G, Comba B, Mira JP: The importance of Toll-like receptor 2 polymorphisms in severe infections. Clin Infect Dis 2005, 4 I Suppl 7:S408-I5.

9. Shuto T, Xu H, Wang B, Han J, Kai H, Gu XX, Murphy TF, Lim DJ, Li JD: Activation of NF-kappa B by nontypeable Hemophilus influenzae is mediated by toll-like receptor 2-TAKI-dependent NIK-IKK alpha /beta-I kappa B alpha and MKK3/6-p38 MAP kinase signaling pathways in epithelial cells. Proc Nat Acad Sci U S A 200I, 98:8774-8779.

10. Shuto $T$, Imasato $A$, Jono $H$, Sakai $A, X u H$, Watanabe $T$, Rixter DD, Kai H, Andalibi A, Linthicum F, Guan YL, Han J, Cato AC, Lim DJ, Akira S, Li JD: Glucocorticoids synergistically enhance nontypeable Haemophilus influenzae-induced Toll-like receptor 2 expression via a negative cross-talk with p38 MAP kinase. J Biol Chem 2002, 277: I7263-17270.

II. Sakai A, Han J, Cato AC, Akira S, Li JD: Glucocorticoids synergize with IL-I beta to induce TLR2 expression via MAP Kinase Phosphatase-I-dependent dual Inhibition of MAPK JNK and p38 in epithelial cells. BMC Mol Biol 2004, 5:2

12. Watanabe T, Jono H, Han J, Lim DJ, Li JD: Synergistic activation of NF-kappaB by nontypeable Haemophilus influenzae and tumor necrosis factor alpha. Proc Natl Acad Sci U S A 2004 I0I:3563-3568.

13. Park JM, Ng VH, Maeda S, Rest RF, Karin M: Anthrolysin $\mathrm{O}$ and other gram-positive cytolysins are toll-like receptor 4 agonists. JExp Med 2004, 200: $1647-1655$

14. Ha U, Lim JH, Jono H, Koga T, Srivastava A, Malley R, Pages G, Pouyssegur J, Li JD: A novel role for IkappaB kinase (IKK) alpha and IKKbeta in ERK-dependent up-regulation of MUC5AC mucin transcription by Streptococcus pneumoniae. J Immunol 2007, 178:1736-1747.

15. Lim JH, Stirling B, Derry J, Koga T, Jono H, Woo CH, Xu H, Bourne $P$, Ha UH, Ishinaga $H$, Andalibi A, Feng $X H$, Zhu $H$, Huang $Y$, Zhang W, Weng X, Yan C, Yin Z, Briles DE, Davis RJ, Flavell RA, Li JD: Tumor suppressor CYLD regulates acute lung injury in lethal Streptococcus pneumoniae infections. Immunity 2007, 27:349-360.

16. Schmeck B, Huber S, Moog K, Zahlten J, Hocke AC, Opitz B, Hammerschmidt S, Mitchell T], Kracht M, Rosseau S, Suttorp N, Hippenstiel S: Pneumococci induced TLR- and Racl-dependent NFkappaB-recruitment to the IL-8 promoter in lung epithelial cells. Am J Physiol Lung Cell Mol Physiol 2006, 290:L730-L737.

17. Yoshida H, Jono H, Kai H, Li JD: The tumor suppressor cylindromatosis (CYLD) acts as a negative regulator for toll-like receptor 2 signaling via negative cross-talk with TRAF6 AND TRAF7. J Biol Chem 2005, 280:4 I I I-4 I I I I

18. Jono H, Lim JH, Chen LF, Xu H, Trompouki E, Pan ZK, Mosialos G, L JD: NF-kappaB is essential for induction of CYLD, the negative regulator of NF-kappaB: evidence for a novel inducible autoregulatory feedback pathway. I Biol Chem 2004, 279:3617|-36174.

19. Lim JH, Jono $\mathrm{H}$, Koga $\mathrm{T}$, Woo $\mathrm{CH}$, Ishinaga $\mathrm{H}$, Bourne $\mathrm{P}, \mathrm{Xu} \mathrm{H}, \mathrm{Ha}$ $\mathrm{UH}, \mathrm{Li}$ JD: Tumor Suppressor CYLD Acts as a Negative Regulator for Non-Typeable Haemophilus influenza-Induced Inflammation in the Middle Ear and Lung of Mice. PLoS ONE 2007, 2: el032.
20. Albiger B, Dahlberg S, Sandgren A, Wartha F, Beiter K, Katsuragi H, Akira S, Normark S, Henriques-Normark B: Toll-like receptor 9 acts at an early stage in host defence against pneumococcal infection. Cell Microbiol 2007, 9:633-644.

21. Lee KS, Scanga CA, Bachelder EM, Chen Q, Snapper CM: TLR2 synergizes with both TLR4 and TLR9 for induction of the MyD88-dependent splenic cytokine and chemokine response to Streptococcus pneumoniae. Cell Immunol 2007, 245: I03-I I0.

22. Mogensen TH, Paludan SR, Kilian M, Ostergaard L: Live Streptococcus pneumoniae, Haemophilus influenzae, and Neisseria meningitidis activate the inflammatory response through Toll-like receptors 2,4 , and 9 in species-specific patterns. Leukoc Biol 2006, 80:267-277.

23. Branger J, Knapp S, Weijer S, Leemans JC, Pater JM, Speelman P, Florquin S, van der Poll T: Role of Toll-like receptor 4 in grampositive and gram-negative pneumonia in mice. Infect Immun 2004, 72:788-794.

24. Malley R, Henneke P, Morse SC, Cieslewicz MJ, Lipsitch M, Thompson CM, Kurt-Jones E, Paton JC, Wessels MR, Golenbock DT: Recognition of pneumolysin by Toll-like receptor 4 confers resistance to pneumococcal infection. Proc Natl Acad Sci U S A 2003. 100:1966-1971.

25. Srivastava A, Henneke P, Visintin A, Morse SC, Martin V, Watkins C Paton JC, Wessels MR, Golenbock DT, Malley R: The apoptotic response to pneumolysin is Toll-like receptor 4 dependent and protects against pneumococcal disease. Infect Immun 2005, 73:6479-6487.

26. Shoma S, Tsuchiya K, Kawamura I, Nomura T, Hara H, Uchiyama R, Daim S, Mitsuyama M: Critical involvement of pneumolysin in production of interleukin-Ialpha and caspase-I-dependent cytokines in infection with Streptococcus pneumoniae in vitro: a novel function of pneumolysin in caspase- $I$ activation. Infect Immun 2008, 76: I547-I557.

27. Moens L, Verhaegen J, Pierik M, Vermeire S, De Boeck K, Peetermans WE, Bossuyt X: Toll-like receptor 2 and Toll-like receptor 4 polymorphisms in invasive pneumococcal disease. Microbes Infect 2007, 9:15-20.

28. van Rossum AM, Lysenko ES, Weiser JN: Host and bacterial factors contributing to the clearance of colonization by Streptococcus pneumoniae in a murine model. Infect Immun 2005 73:7718-7726.

29. Preston JA, Beagley KW, Gibson PG, Hansbro PM: Genetic background affects susceptibility in nonfatal pneumococcal bronchopneumonia. Eur Respir J 2004, 23:224-231.

30. Gingles NA, Alexander JE, Kadioglu A, Andrew PW, Kerr A, Mitchell TJ, Hopes E, Denny P, Brown S, Jones HB, Little S, Booth GC, McPheat WL: Role of genetic resistance in invasive pneumococcal infection: identification and study of susceptibility and resistance in inbred mouse strains. Infect Immun 200I, 69:426-434

3I. Massoumi R, Chmielarska K, Hennecke K, Pfeifer A, Fassler R: Cyld inhibits tumor cell proliferation by blocking Bcl-3-dependent NF-kappaB signaling. Cell 2006, I 25:665-677.

32. Zhang J, Stirling B, Temmerman ST, Ma CA, Fuss IJ, Derry JM, Jain A: Impaired regulation of NF-kappaB and increased susceptibility to colitis-associated tumorigenesis in CYLD-deficient mice. J Clin Invest 2006, I I 6:3042-3049.

33. Reiley WW, Zhang M, Jin W, Losiewicz M, Donohue KB, Norbury CC, Sun SC: Regulation of T cell development by the deubiquitinating enzyme CYLD. Nat Immunol 2006, 7:4 I I-4I7.

34. Reiley WW, Jin W, Lee AJ, Wright A, Wu X, Tewalt EF, Leonard TO, Norbury CC, Fitzpatrick L, Zhang M, Sun SC: Deubiquitinating enzyme CYLD negatively regulates the ubiquitin-dependent kinase Takl and prevents abnormal $\mathbf{T}$ cell responses. J Exp Med 2007, 204: |475-|485.

35. Sakai A, Koga T, Lim JH, Jono H, Harada K, Szymanski E, Xu H, Kai H, Li JD: The bacterium, nontypeable Haemophilus influenzae, enhances host antiviral response by inducing Toll-like receptor 7 expression: evidence for negative regulation of host anti-viral response by CYLD. Febs J 2007, 274:3655-3668.

36. Berry AM, Lock RA, Hansman D, Paton JC: Contribution of autolysin to virulence of Streptococcus pneumoniae. Infect Immun 1989, 57:2324-2330.

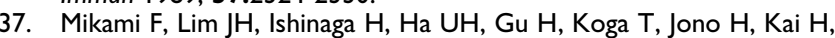
Li JD: The transforming growth factor-beta-Smad3/4 signaling pathway acts as a positive regulator for TLR2 induction 
by bacteria via a dual mechanism involving functional cooperation with NF-kappaB and MAPK phosphatase I-dependent negative cross-talk with p38 MAPK. J Biol Chem 2006, 28 I:22397-22408.

Publish with Bio Med Central and every scientist can read your work free of charge

"BioMed Central will be the most significant development for disseminating the results of biomedical research in our lifetime. " Sir Paul Nurse, Cancer Research UK

Your research papers will be:

- available free of charge to the entire biomedical community

- peer reviewed and published immediately upon acceptance

- cited in PubMed and archived on PubMed Central

- yours - you keep the copyright

Submit your manuscript here:

http://www.biomedcentral.com/info/publishing_adv.asp
BioMedcentral 